\title{
Spanish regulatory approach for Biobanking
}

\author{
Javier Arias-Diaz ${ }^{\star,}$, María C Martín-Arribas ${ }^{1}$, Javier García del Pozo ${ }^{1}$ and Carlos Alonso ${ }^{2}$
}

The Spanish regulatory framework for storage of samples for research responds to most issues raised by both researchers and society regarding biobanking. The Spanish regulation currently foresees three possible ways in which these samples are to be handled: (a) gathering for use in a specific project, (b) storage in a collection, and (c) storage in a Biobank. Samples incorporated into a 'collection' can only be used by the investigator who requested them and cannot be transferred to third parties or used in research projects outside the particular research line foreseen in the original consent. On the other hand, the legal entity 'Biobank' refers not only to a set of physical facilities but to the management of the samples stored under that label, and particularly to the requirements for their cession. An approach based on putting most of the regulatory weight on the biobank side has been chosen in order to guaranty the rights of the donors as well as to ease the task of the researchers. A Biobank requires both to be authorized and to be registered in a public Registry. The requirements are quite stringent, allowing for the consent to be given as 'broad' in scope without implying being 'blank.' In this regard, for Biobanks to justify the taking on of some of the donors' rights, a key requirement is to have an external ethics committee supervising the adequacy of samples cession and use, notwithstanding the need for a previous bioethical supervision of the target protocol. European Journal of Human Genetics (2013) 21, 708-712; doi:10.1038/ejhg.2012.249; published online 28 November 2012

\section{INTRODUCTION}

It is generally accepted, nowadays, that the search for excellence in biomedical research requires the use of tissue samples and data from humans. Interestingly, in recent years this type of research is increasingly focusing on testing scientific hypotheses through the use of in vitro studies on human samples. In fact, in hospitals, several pathological conditions have been described by analysis of stored clinical tissue specimens, bringing results of great scientific and practical relevance. Obviously, the availability of high-quality samples and clinical data and the associated socio-demographic aspects of these samples are key aspects to take into account when the performance of translational research is considered. ${ }^{1}$

It is known, however, that the use of human samples for research purposes raises ethical question marks. In fact, several developments in the field of molecular biology and the development of new technologies have greatly facilitated the access to genetic information. Today, human samples are seen to be carriers of data that may determine the predisposition to certain diseases not only of the donor subject but also of their relatives. ${ }^{2}$ Thus, the same general principles applied to personal data protection must be implemented when using those samples.

Although in Spain there is abundant legislation on biomedical research, particularly related to drug clinical trials, regulation concerning the collection, storage, and the use of human samples for research was lacking. In spite of the fact that the storage of samples for research purposes had been carried for a long time before, the legislation on these matters in Spain is quite recent. In July 2007, the Biomedical Research Act (BRA) was released ${ }^{3}$ in an attempt to respond to some previously identified shortfalls. The BRA has found a further development in the 1716/2011 Royal Decree that has come into force on June 2012. ${ }^{4}$ The Royal Decree defines the basic requirements for authorization and operation of Biobanks for biomedical research and also creates a National Registry of Biobanks. With the entry into force of the Spanish latest piece of regulation, the use, collection and transfer of samples for research purposes is fully configured. The major object of this regulation frame is to ensure that the gathering, storage, and cession of human samples are carried out under appropriate quality settings and ethical requirements.

The Spanish regulation offers novel approaches to already acknowledged biobanking dilemmas. Its innovative character warrants making a review of its content and practical implications.

\section{BIOBANKS VERSUS COLLECTIONS}

The Spanish regulation currently foresees three possible ways in which samples for research are to be handled:

- Gathering for use in a specific project

- Storage in a collection

- Storage in a Biobank

Samples gathered for a specific project are time-restricted, having to be destroyed at the end of that particular project. If a new use of the samples arises after the completion of the research, the leftovers have to be integrated into a collection or a Biobank, provided that the donor is approached to obtain the appropriate consent.

For long-term storage of samples devoted to be used within the frame of a particular research line, there is a specific legal entity herewith termed 'collection.' A 'collection' is defined as 'an orderly and permanent set of human biological samples that are stored outside the Biobank regime' as defined below. These samples can only 
Table 1 Possible regimes for the use of human samples for research

\begin{tabular}{|c|c|c|}
\hline Specific Project & Collection & Biobank \\
\hline $\begin{array}{c}\text { National Registry } \\
\text { Not required }\end{array}$ & \multicolumn{2}{|c|}{$\begin{array}{c}\text { National Registry } \\
\text { Required }\end{array}$} \\
\hline $\begin{array}{c}\text { Ethical Assessment } \\
\text { REC }\end{array}$ & $\begin{array}{c}\text { Ethical Assessment } \\
\text { REC }\end{array}$ & $\begin{array}{c}\text { Ethical Assessment } \\
\text { REC and Biobank ethical } \\
\text { committee }\end{array}$ \\
\hline $\begin{array}{l}\text { Administrative } \\
\text { authorization } \\
\text { Not required }\end{array}$ & $\begin{array}{l}\text { Administrative } \\
\text { authorization } \\
\text { Not required }\end{array}$ & $\begin{array}{l}\text { Administrative } \\
\text { authorization } \\
\text { Required }\end{array}$ \\
\hline $\begin{array}{c}\text { Scope of the consent } \\
\text { Particular project }\end{array}$ & $\begin{array}{l}\text { Scope of the consent } \\
\text { Particular project / } \\
\text { research line }\end{array}$ & $\begin{array}{l}\text { Scope of the consent } \\
\text { Any research, with } \\
\text { possible restrictions }\end{array}$ \\
\hline $\begin{array}{c}\text { Third party use } \\
\text { No }\end{array}$ & $\begin{array}{c}\text { Third party use } \\
\text { No }\end{array}$ & $\begin{array}{c}\text { Third party use } \\
\text { Yes }\end{array}$ \\
\hline
\end{tabular}

be used by the investigator who requested them and cannot be transferred to third parties or used in research projects outside the particular research line foreseen in the original informed consent. This does not imply that collections cannot be physically stored in the Biobank facilities.

The regime applicable to Biobanks is different from that of collections, because the primary objective of Biobanks is to serve as platforms to provide the scientific community with high-quality samples. To an accurate understanding of the term 'Biobank' it is relevant to point out that it refers not only to the physical facilities of the Biobank but, above all, to the management of the samples stored under that label, and particularly to the requirements for their cession (Table 1).

Samples obtained for therapeutic or diagnostic purposes will be affected by this normative in case they were going to be used for a biomedical research. This aspect is particularly important because it implies that any collection of samples obtained for care (eg, those stored in the pathology department of a hospital) must eventually be adapted to comply with the BRA regulation. In fact, in Academic institutions research using these samples would be quite common. This implies that most pathology departments should either set up a Biobank or be ascribed to one. As the samples stored in any of those departments are usually counted by tens of thousands, it is unlikely that all of them simultaneously enter into Biobanks. It is rather expected that the incorporation of the samples into Biobanks gradually goes on as they are specifically requested by the researchers involved in particular projects.

In any case, the primary use of the sample for diagnostic or therapeutic purposes must be assured. To avoid the real possibility that the use in research results in insufficient remaining material for that primary use, the professional responsible for these uses will be in charge of its priority allocation.

\section{ESTABLISHMENT AND FUNCTIONING OF BIOBANKS}

The minimum requirements to grant an authorization leading to the establishment and functioning of a Biobank are the following:

- That the organization, objectives, and available resources justify the Biobank usefulness for research.

- That both a scientific director of the Biobank and a person responsible for the personal data file have been appointed.
- That two external committees, a scientific and an ethical one, have been assigned to the Biobank.

- That the Biobank activity is not entailed for profit.

- That the data file has been registered in the General Registry of the Spanish Agency of Data Protection.

- That the facilities and resources necessary to ensure quality preservation of the samples are operative.

The authorization for the establishment and operation of a Biobank corresponds to the competent authority of the Autonomous Community where it is established. National Biobanks are also foreseen that will be created by the Central Governmental Administration. In principle, the authorization is granted for an indefinite period of time although any substantial modification requires prior authorization.

\section{ORGANIZATION OF BIOBANKS}

The Spanish regulation endows the Biobanks with a defined structure to ensure compliance with the quality, legal, and ethical requirements. Accordingly, a Biobank should have at least: (a) a title holder, which can be a person or an institution and (b) a management structure made up by a Scientific Director, a person responsible for the personal data file, and two committees of external experts, a scientist one and an ethical one. A Biobank can also function as a network, with a centralized management structure and a decentralized activity. In this case, a person in charge should be appointed for each Biobank hub.

Each one of the external committees is to be made up by a minimum of four members with knowledge in matters relating to the functions they are committed to. The members must not have any direct participation in the activity of the Biobank and should abstain if any conflict of interest arises.

To qualify as an 'external committee' none of the members can be part of the structure of the Biobank or have any conflict of interest with the activity of the Biobank. The essential role of both committees will be to report on the ethical and scientific aspects of the incorporation of existing sample collections to the Biobank and to report on the transfer of samples to other Biobanks or to research groups. As a guarantee of transparency, the Biobank has the obligation of publishing the identity of the members of the external committees. From a practical point of view, existing Research Ethics Committee (REC) of the institution that hosts the Biobank might assume the role of the Biobank external ethics committee.

\section{NATIONAL REGISTRY OF BIOBANKS}

A remarkable feature of the Spanish regulation regarding the storage of human samples for research is the creation of the National Registry. All collections and Biobanks must be registered, and the Registry will be made publically available. This way, RECs members, when evaluating a research project involving the use of human stored samples, can trace the origin of the samples while considering consent-related issues. The Carlos III Institute of Health is entitled for the maintenance of the National Registry.

\section{INFORMED CONSENT}

The compilation, storage, and use of samples for research purposes requires the prior consent of the donor subject for its use in a particular research project, manifested upon the receipt of appropriate information. The need of prior consent is independent on whether the samples are intended for long-term storage or short-term use, or to be handled in an anonymous or coded way.

The procurement of an informed consent is a process that is finally expressed in a document containing both the information leaflet and 
an agreement sheet that has to be signed by the donor. The information leaflet should include the principal investigator contact data. Possible options for the samples destination (particular project, storage as a collection, or Biobank) must be shown separately in the agreement sheet. This way the donor will have the opportunity to select any purpose in an independent manner.

\section{INCORPORATION OF SAMPLES INTO BIOBANKS}

Taking into account whether the samples were obtained before the BRA or at a later stage, and the extent of the given consent, several possibilities are to be considered:

\section{Samples obtained before the entry into force of the BRA}

(a) If there is an informed consent for research, even if it is unspecific, samples can be incorporated into the Biobank regime for their use by third parties for the purposes specified in the informed consent.

(b) If there is no informed consent, they may be used for research when getting the consent represents an unreasonable effort, or if it is not possible at all because the donor had died or were untraceable.

In these cases, the use of the samples requires the favorable opinion of the REC after considering at least the following requirements:

- That the research is of public interest.

- That the research is less effective or not possible without the identification data of the donor.

- That there is no known objection to the use of the samples.

- That the confidentiality of the donor's personal data is ensured

(c) If the samples are anonimyzed (irreversible dissociation), no further requirement will be needed to incorporate them into the Biobank regime.

\section{Samples obtained after entry into force of the BRA}

(a) If an informed consent is lacking or there is a generic consent for research.

In this case, the only option for their use is to seek the consent of the donor for storage of the samples in a Biobank.

(b) If consent is available for use in a specific project or research line.

Regardless that the samples are physically stored in a Biobank facility or not, the samples can only be used for that specific project or research line. If the samples are to be used in a different project or be used by other researchers, either a new consent should be obtained for those purposes or to become integrated into a Biobank. For that latter purpose, a new consent should also be obtained (Figure 1).

In all cases, the informed consent document must include the possibility of introducing restrictions to the use of the samples. These restrictions should be respected.

\section{SAMPLES FROM DECEASED PERSONS}

Both the BRA and the Royal Decree foresee the procurement and storage of biological samples from deceased persons for research. The gathering of samples is allowed to be done when the deceased person had manifested his/her consent in life or at least there is no known expressed opposition to the use of the samples. In this latter case, the family cannot object without a reasoned plea. In any case, efforts to gather information about the will of the deceased person should be made. The existence of advance directives will be explored and, in the absence of these, near family members of the deceased person and the attending health care workers will have to be consulted regarding the donor's will. That consultation will also have to be documented.

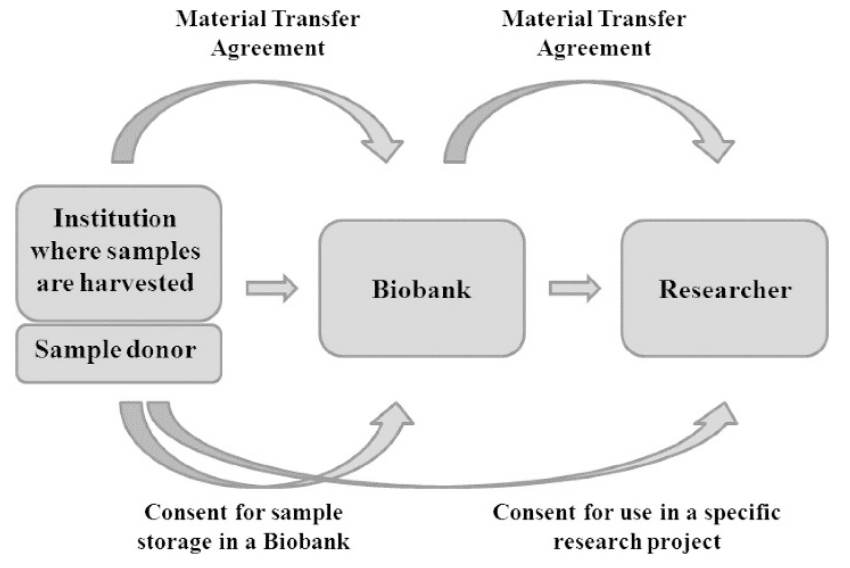

Figure 1 Donor-specific consent requirement. Notwithstanding the need of a written agreement for sample transfer, a specific consent of the donor for its destiny is always required.

The report of the REC will be always required. The family members retain the freedom to request the withdrawal of the already stored samples or their irreversible anonymization, provided that this request is made on a justified basis.

An additional requirement for collecting those samples is the existence of a prior documented agreement between the researcher or the Biobank, on the one hand, and the center responsible for the custody of the corpse, on the other, whether it be a hospital, a provider of funeral services, or the forensic anatomical institute.

\section{DISCUSSION}

The Spanish management model of human biological samples for research aims to facilitate researchers the access to the largest possible number of samples that fulfill the prerequisites of quality, gratuity, and transparency. These features will facilitate the development of research projects of high quality together with a suitable respect for the wishes of donors. Unlike other countries in which the regulation has been originated from the need to create biobanks to support large-scale projects, Spain has had as a priority to regulate the handling of already existing large collections of biological samples scattered through the network of the National Health Service hospitals, public research institutions, and universities. As a consequence of that, the Biobank legal entity has been created.

The regulation of biobanks and the use of human tissues and samples for research vary reacting to specific legal and ethical traditions. Some countries have regulated the use of human tissues and biobanks for research by means of a discrete Law. Spain has been one of the pioneers in taking this approach. Other countries have addressed these issues through a rather fragmented jurisdiction, entailing references to the Civil and/or Penal Code, Constitutional law, Codes of professional conduct, transplantation-, transfusion-, funeral-, and drug- as well as data protection laws. The countries most recently adhered to the $\mathrm{EU}$ and candidate states are using transnational directives and codes, like the EU Directives (eg, the Tissue Directive ${ }^{5}$ ), Recommendation on research on biological materials of human origin ${ }^{6}$ or the Oviedo Convention, ${ }^{7}$ as guidance.

The complexity of regulation comes mainly from the need to deal with sensitive issues such as the legal status of the samples, the informed consent, or the need to guarantee donors' privacy. One of the most controversial issues is the right of the donor to be informed about the results of research, being especially relevant in cases of 
incidental findings and/or when the samples come from large-scale Biobanks. $^{8}$

While most countries are reluctant to grant donors property rights of the samples, in Spain, Germany, and Portugal donors maintain actual ownership of their samples. The role of a Biobank would be to act as a custodian or depositary trustee of the samples ensuring a proper use according to the will of the donor.

RECs from most countries recommend evaluating research protocols on a case-by-case basis. ${ }^{9}$ If a research protocol involves samples coming from several biobanks, not to mention at a transborder level, its ethical assessment can be a daunting task for a REC. The Spanish strategy has been to endow every Biobank with a bounding external ethics committee that supervises the adequacy of the gathering and cession of the samples. This way, the REC assessing a research project, once it has been satisfied that all samples come from accredited/certified biobanks, it could further set aside donor-and-consent-related issues and just focus on the specific aspects of the protocol. The Biobank ethics committee criteria could also be useful for making decisions in complex issues such as what relevant findings/information should be returned to the donor, possible limits of research to be carried out in children samples, or special cases when to seek additional consent from the donor.

Facing the issue of the extent of the donor informed consent, the Spanish approach has been to define a particular regime for biobanks, allowing a certain degree of flexibility to the possible use of the samples, without implying, however, that the informed consent has been given as a 'blank' consent. Instead, the donor gives consent for the storage of the sample in an authorized Biobank, considered to be a somewhat 'controlled' place. We believe that this approach represents something new compared with the handling of biological human samples on biobanks in other European Countries.

A Biobank requires both to be authorized by a competent authority and to be registered in the National Registry of Biobanks. The authorization requirements are quite stringent, allowing, thus, for the consent to be given as 'broad' in scope. In this regard, a key requirement is that a Biobank must have an external dedicated ethics committee that supervises the adequacy of each sample cession, notwithstanding the need for a previous bioethical supervision of the target protocol. Thus, the guarantees provided by the Biobank satisfy demanding ethical requirements while avoiding making the access to the samples unnecessarily difficult and providing legal assuredness to the researcher.

Regarding the protection of data privacy and confidentiality in the context of Biobanks, most countries have their laws transposed from the specific EU directive, ${ }^{10}$ as it is also the case of Spain. The general criteria are that the samples and the associated data that have been stored in Biobanks are to be transferred only in either an anonymous or a dissociated way. However, in cases where the nature of the research project requires additional data of the subject source, the Biobank can coordinate the gathering of this information with the center where the samples were obtained.

The Biobank is also responsible for ensuring that the transfer of the samples and data is done safely. This is especially relevant for transborder flow of specimens. The country or institution receiving the samples must meet the same safety requirements that exist in Spain. Most EU countries include this criterion. Only Estonia having a National Biobank does not allow the transfer of samples to other countries.

A highly discussed issue in biobanking refers to the potential return to the donor of the results of those investigations that have been carried out with his/her samples. The differences are primarily centered on whether the results must be reported to donors in either an individual or an aggregated form, or whether those results just relevant to the donor's health must be returned. The Spanish Law fully recognizes the donor's right to information upon request, whether or not it is relevant for his/her health. In cases of unexpected results that may be relevant to the donor's health, the Biobank ethics committee may decide when to convey individualized information to the subject source. Regarding the return of the results originated from research, the law does not establish any difference whether the samples were stored as a collection or stored in a biobank.

The Law also refers to the requirements that must be considered in relation to the right not to know. On the one hand, limitations to the type of information provided to the family members are established; on the other hand, the convenience that the person concerned, where appropriate, should transmit this information to his family members is also stated. When this information, according to the criteria of the medical staff in charge, is necessary in order to avoid major damage to the donor's health or his relatives, a close family member or a representative shall be informed, after clinical ethics committee consultation.

Due to the above-mentioned Spanish reality of already existing valuable collections of biological samples, difficult to integrate at once in the Biobank regime, it has been considered as necessary to keep a regime of 'collections of samples', somehow parallel to that of the Biobank. This situation may entail the difficulty of defining the scope or the extent of the donor consent for the use of the sample in a particular research line. In certain vanguard research areas, it is becoming increasingly difficult to define their frontiers. It is well known about the complex connection between, for example, cancer and aging or degenerative diseases and basic mechanisms of inflammation. In any case, the responsibility for deciding whether a new consent is to be required lies on the competent REC in charge of evaluating each new project, intended to be framed in that line.

\section{CONCLUSIONS}

The Spanish regulatory framework for storage of samples intended for biomedical research tries to respond to most of the issues raised by both researchers and society regarding biobanking in the modern era.

In order to guaranty the rights of the donors as well as to ease the task of the researchers, an approach based on putting most of the regulatory weight on the biobank side has been chosen. For Biobanks to justify the taking on of some of the donors' rights on the use of their samples, alongside to the need of authorization and administrative supervision, they have to rely on an external ethics committee to take care of most decisions regarding sample and/or information transfer.

The expecting future trend would be for collections to be progressively turning into the Biobank regime to be able to respond to the increasingly higher stringent ethical standards requested by society and to make samples accessible for other researchers' projects.

\section{CONFLICT OF INTEREST}

The authors declare no conflict of interest.

\footnotetext{
1 Martín-Arribas MC, Arias-Díaz J: Biobanks and use of samples of human origin for surgical research. Current regulatory framework. Cir Esp 2011; 89: 207-212.

2 Bosch-Comas A, Morente M: [Importance of biobanks for biomedical development in Spain]. Enferm Infecc Microbiol Clin 2011; 29: 643-644.
} 
3 Act 14/2007, on Biomedical Research. http://www.boe.es/boe/dias/2007/07/04/pdfs/ A28826-28848.pdf (last consulted on 2 August 2012).

4 Royal Decree 1716/2011, on the minimal requirements for authorization of biobanks for biomedical research. http://www.boe.es/boe/dias/2011/12/02/pdfs/BOE-A-2011 18919.pdf (last consulted on 2 August 2012).

5 Directive 2004/23/EC of the European Parliament and of the Council of 31 March 2004 on setting standards of quality and safety for the donation, procurement, testing, processing, preservation, storage and distribution of human tissues and cells Tissue Directive Official J L, 102/48, 7.4.2004 P. 0049 - 0051

6 'RecommendationRec(2006)4 of the Committee of Ministers to member states on research on biological materials of human origin'. (Adopted by the Committee of Ministers on 15 March 2006 at the 958th meeting of the Ministers' Deputies).
7 Convention for the Protection of Human Rights and Dignity of the Human Being with regard to the Application of Biology and Medicine: Convention on Human Rights and Biomedicine. Strasbourg: Council of Europe, 1997, CETS No.: 164.

8 Beier K, Lenk CA: Unified European approach on tissue research and biobanking? A comparisonInLenk C, Sandor J, Gordjin B (eds). Biobanks and tissue research. The public, the patient and the regulation. The International Library of Ethics, Law and Technology. Springer Science \& Business Media: New York, 2011; pp 143-164.

9 Guide for Research Ethics Committee Members. Strasbourg: Council of Europe 2011. http://www.coe.int/t/dg3/healthbioethic/source//NF(2011)_en.pdf (last consulted on 2 August 2012).

10 Directive 95/46/EC of the European Parliament and of the Council of 24 October 1995 on the protection of individuals with regard to the processing of personal data and on the free movement of such data Official JL, 281, 23.11.1995 P $0031-0050$. 